\title{
The Efficiency of Discrete Event Systems for the General Pickup and Delivery Problem with Electric Vehicles
}

\author{
AbdelhakOutalha $^{1 *}$, Yassine Lakhal ${ }^{1}$, Fatima Zahra Baghli $^{1}$ and Fouzia Kzaiber ${ }^{1}$ \\ ${ }^{1}$ Laboratory of Engineering and Applied Technologies (LITA), Hight School of Technologie, Sultan \\ Moulay Slimane University, Beni Mellal, Morocco
}

\begin{abstract}
Transport on-demand is an issue in the Vehicle Routing Problem which involves transporting a collection of goods from the source (depot) to their destination (customers) and inversely. This paper analyzes and forecasts the viability of using discrete event systems in a subset of this general problem known as pickup and delivery problems in the literature and projects on an automated manufacturing cell with an optimization approach for the vehicle's energy consumption. Keywords: Discrete Event Systems, General Pickup and Delivery Problem, Electric Vehicles, Modelling.
\end{abstract}

\section{Introduction}

Logistics companies typically use the Vehicle Routing Problem (VRP), which aims at reducing transport costs for visiting customers, because each customer is exactly visited once and every path begins and ends in one depot. Dantzig and Ramser [1] developed the original VRP. Besides, several VRP variations and extensions have been introduced over the years to incorporate real-world limitations and conditions. The Capacitated VRP (CVRP) method [2], in which cars have restricted freight power, and the VRP with time windows (VRPTW) in which customers must be reached within a specific, fixed period $[3,4]$, are two of the most widely studied extensions. It is commonly recognized that the entire range of vehicle routing problem can be divided into two, nodes routing problem of which the TSP (Traveling Salesman Problem [5]) and the GPDP (General Pickup and Delivery Problem [6]) belong, and arcs routing problem, of which the best known are CPP (Chinese Postman Problem [7]) and the CARP (Capacitated Arc Routing Problem [8]). This classification is presented in figure 1 .

* Corresponding author: abdelhak.outalha.doc@gmail.com 


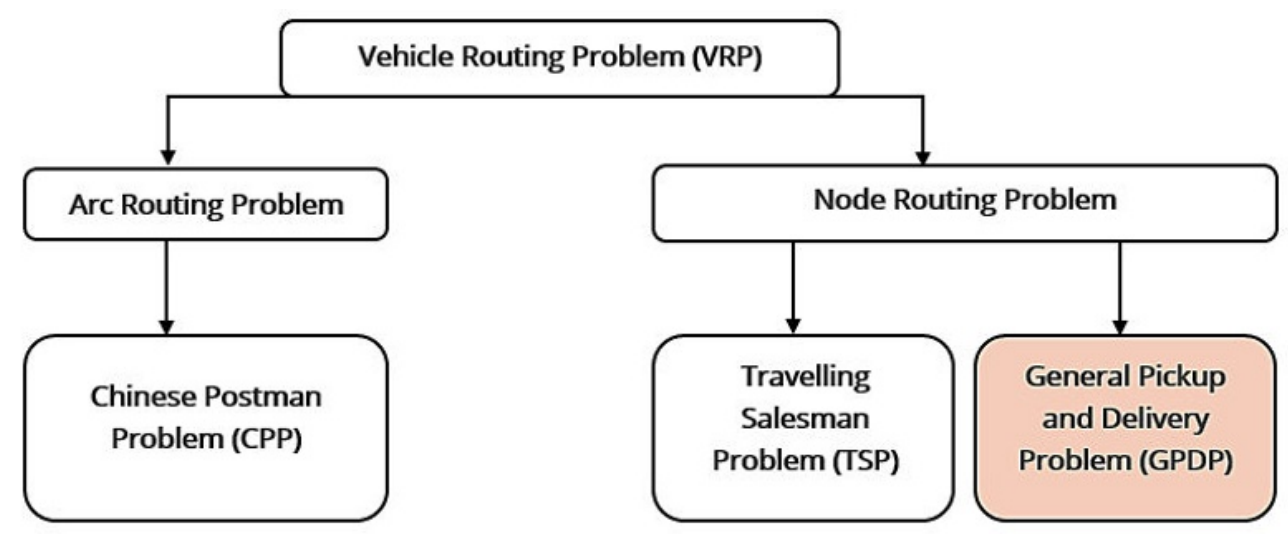

Fig. 1. Family of Vehicle Routing Problem.

In this paper, we introduce the pickup and delivery problem with electric vehicles using discrete event systems. This work completes our first VRP research [10], in which we have given the opportunity to model with Petri Nets. The remainder of the paper is structured as follows. Section (2) presents the literature Review of the problem statement. The proposed modelling approach using discrete event systems is presented in section (3). Tour optimization approach and first results are reported in section (4). Finally, in section (5), a conclusion and perspectives are given.

\section{Literature Review of General Pickup and Delivery Problem}

The In this part, we briefly review the literature related to the problem studied in this paper. The family of General Pickup and Delivery Problem (GPDP) is described by (Savelsbergh and Sol, 1995) [6]. More recently, the state of the art (Berbeglia et al., 2007) [9] can also be cited. It is a family of problems where it is not sufficient simply to visit the different summits with the vehicles, but where the nature of the requests must also be taken into account. These requests concern a set $X=\left\{x_{i}\right\}$ of product types. Requests may be of two distinct types: a pickup request or a delivery request. A request is aligned with a summit $\mathrm{j}$ of the graph and involves the number $\mathrm{q}_{\mathrm{ji}}$ of product $x_{i}$. The graph compares a request for pickup with a positive quantity of $\mathrm{q}_{\mathrm{j} i}>0$ while a request for delivery with a negative quantity of $\mathrm{q}_{\mathrm{ji}}<0$. The perfect solution for such a problem is a sequence of tours including: all demands are met, the full vehicle load at all stages of the tour is respected and the net tour expenses are low. Vehicle routing problem are generally modelled on a graph oriented $G=$ $(V, E)$, with $V=\{1, \ldots, n\}$ the set of summits that model the requests. To this range of summits $\mathrm{V}$ is added a summit of 0 correspondings to the depot of the vehicle fleet. $E=\{(i$, $j) \mid i, j \in V\}$ is the set of arcs. To a summit $i$, is associated with a set of values $\mathrm{q}_{\mathrm{ji}}$, which correspond to the demand for $x_{j}$ on the summit $i$. To an arc $(i, j) \in A$ is associated with a value $C_{i j}$ which corresponds to the cost of going from the summit $i$ to $j$. Figure 2 provides an example of a solution composed of one tour. The vehicle shall be assigned to the tour and a tour shall be processed by an ordered customer's sequence. It begins with the depot, attends the ordered customers list and returns to the depot. 


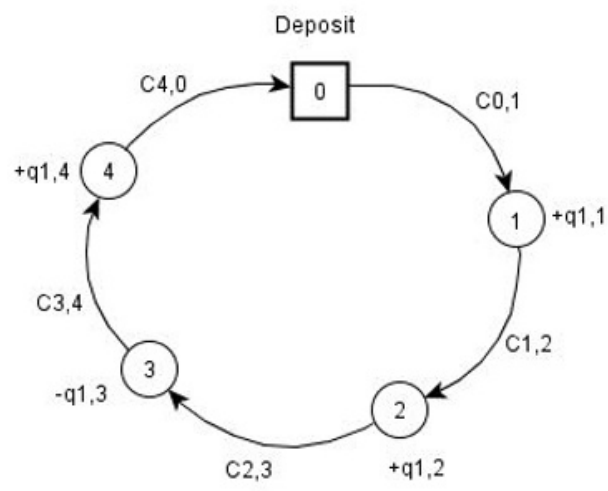

Fig. 2. Example of a solution composed of one tour, one vehicle and 4 customers.

This family of problems (GPDP) has two necessary attributes: the structure and the form of visits which must be made. For the structure, the system includes the management of summits for pickup and delivery, wish is typically divided into three distinct forms, as seen in Figure 3, arcs are not vehicles rounds but transport links that are to be formed between pickup locations and goods delivery. Each arc corresponds to a products category $x_{i}$. The continuous arc is the product $\mathrm{x}_{1}$, and the dashed arc is the products $\mathrm{x}_{2}$ and $\mathrm{x}_{3}$.

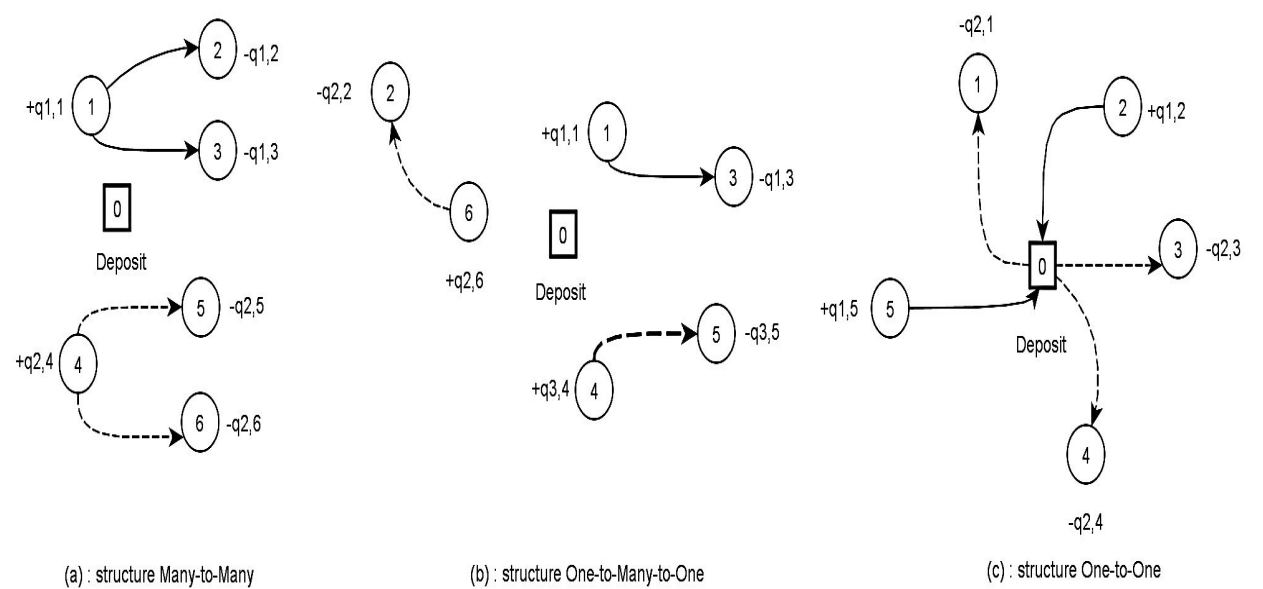

Fig. 3. Possible Structures for GPDP.

The first structure, figure 3 (a) is called Many-to-Many (M-M) and models problems for which there are multiple pickup summits and multiple delivery summits for each type of object $x i$. The second structure, figure 3 (b) is called One-to-Many-to-One (1-M-1), in which the depot includes the products for pickup as well as for delivery. The third figure 3 (c), called One-to-One (1-1), models problems where each pickup request corresponds to a 
single delivery request. The second part refers to the existence of the vehicle's access to graph summits. A summit may have different characteristics in GPDP problems, it can be connected only to requests for pickup or delivery or both, pickup and delivery at the same time. Subsequently, many constraints will enrich the GPDP problems, for example, the problem with time windows, which allows that one or more problem requests be handled with an interval to begin vehicle processing immediately, as presented in [11], this study proposes a new exact PDPTW algorithm based on a fixed partitioning, and [12] suggests a metaheuristic based on the simulated annealing algorithm which restarts a search process after several non-improved iterations from the current best solution. There is also the problem with multi-depot [13], this study shows the efficiency of the ALNS approach, and the problem with heterogeneous vehicle fleets where the fleet does not consist of the same vehicles, such as in [14], a Grouping Genetic Algorithm (GGA) is proposed. We can also note the problem of maximum vehicle travel time. This restriction requires a cumulative period for the rounds of a solution, which is the difference between the date of departure from the depot and the date of the return to the depot, it can reflect vehicle autonomy, for example in the case of electric vehicles as it is studied in [15]. Finally, the problem of the maximum transport time for goods. This restriction is called the maximum transit time in the literature, is also used to model customer service quality [16]. To solve these Vehicle Routing Problems, like GPDP, various types of approaches are possible. As seen in Figure 4 , the current strategies are summarized.

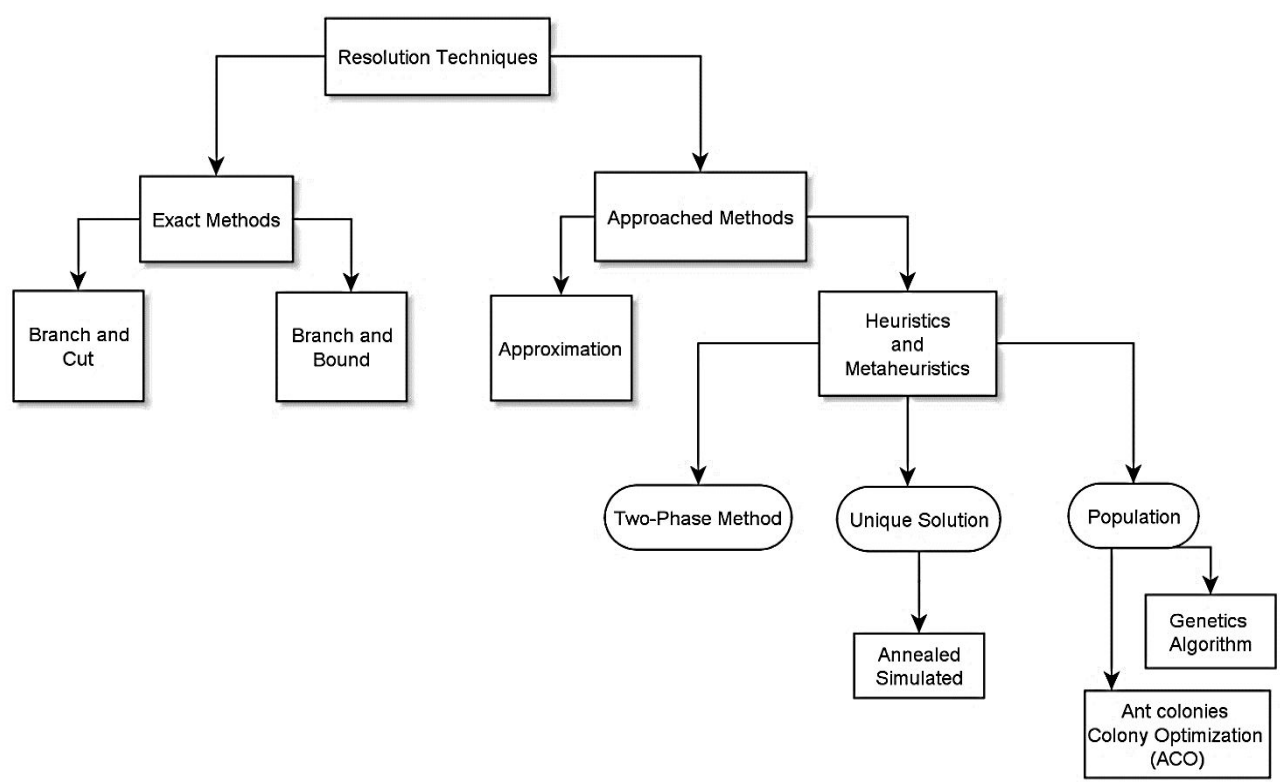

Fig. 4. Approaches used for Vehicle Routing Problem.

These methods can be divided into two: exact methods and approached methods. Exact methods are efficient in small cases and precise algorithms, also are based on formulations of mathematical problems. However, these methods do not offer remedies for medium or wide instances promptly. In general, it can be divided into several categories, as defined in [17]. There are two types of so-called branching methods: Branch and Bound, Branch and Cut. Both approaches are based on the separation and evaluation theory. Approached 
methods permit the fast processing of large instances. The solutions found are generally of high quality but can not be refined; these approached methods can be divided into two: approximation methods and heuristic methods. Approximation methods lead to solutions which have not been optimally tested, and heuristic/metaheuristic methodologies are ways for obtaining high-quality solutions in a limited period of time. The variance between the two methods means that a heuristic has a particular optimization problem when a metaheuristic is a general algorithm that can be used for multiple applications problems in optimization.

\section{Discrete Event Systems methodology for GPDP}

As explained and elaborated in [18], discrete event systems (DES) constitute complex, isolated and fastly developing state-space structures induced by physical events, the motion of the states is constant in parts. Related technologies help define and design various processes, including modular production systems, IT architectures, communications and transport networks. All of these systems adopt operating principles, or algorithms, which adjust as a result of discrete asynchronous events over time as an arrival of a message, order for execution or acquisition of a task. Modelling is generally done by the formal language, automata, and petri nets. In this part we have thought about projecting our problem (GPDP) on [19], this work addresses the problem of routing control in an automated manufacturing cell that consists of three work stations, a transportation system, a feeding conveyor and a retrieving conveyor as shown in figure 5 .

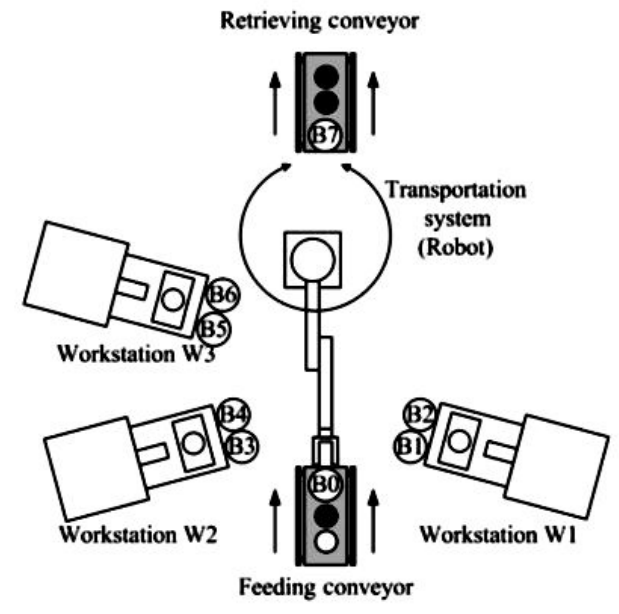

Fig. 5. Example of automated manufacturing cell.

Return to our topic GPDP, this problem based on the number of customers. Mathematical calculations are typically used in modeling regarding cost optimization, which ensures that the energy consumption of any automotive type can be reduced, either on a standard basis for combustion engines or on electric engines with hybrids and pure electric vehicles. Minimize travel time to complete a tour as well. We suggested in [10] the petri nets that modelling this problem can be carried out graphically. These nets are an effective method for modeling the complex structures, they are based on the design of the structure studied 
and provide the possibility to use multiple models. As the concept of using colored petri nets, the model is flexible and easier to understand, by offering each customer a color to represent him. Our idea is therefore to consider that the transport system is the electric vehicle and the workstations are the customers and charging stations using time-delayed state machines. The alphabet $\{\mathrm{a}, \mathrm{b}, \mathrm{c}, \mathrm{d}\}$ with each letter corresponding to a distance, is given by using the formal language and crossing the move can only be achieved if and only if the distance is extinguished, and the alphabet size is equal to the number of customers. As shown in figure 6 the distribution of 3 customers is given.

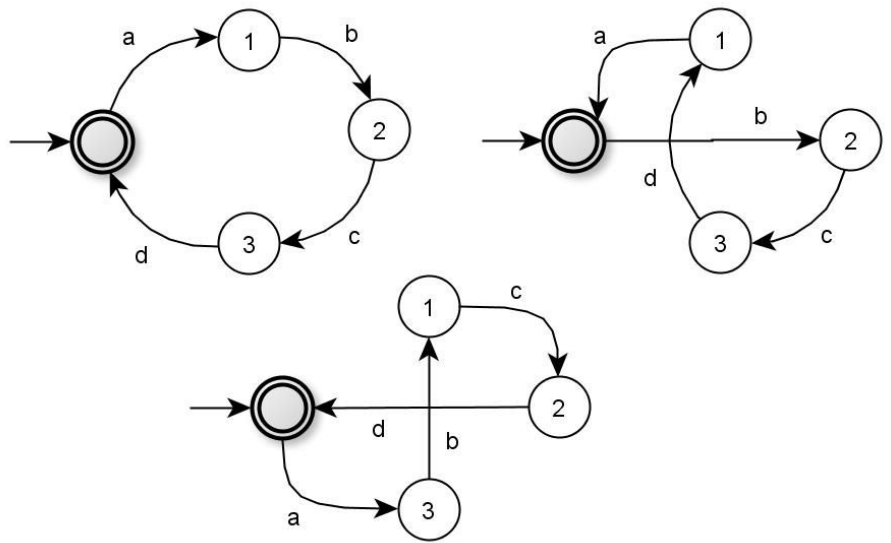

Fig. 6. Example of distribution for 3 customers.

The Three stations 1, 2 and 3 (nodes) are the customers and the transition from one station to the next reflects the electric vehicle.

\section{Tour Optimization Approach}

This section describes the tour optimization approach. The problem formulation is started at the customer's request, and this request must include at least 2 customers. Distances will then be established. These distances are split into two, the distance between the depot and each customer and the distance between customer $<\mathrm{i}>$ and customer $\langle\mathrm{j}\rangle$. Once the number of requests is known, customer's ranking will be considered to make the tour optimal. As seen in the diagram in Figure 7, the state of charge of the battery will be taken into account to reduce the tour's overall consumption. As the vehicle arrives on customer $<$ $\mathrm{i}>$, the estimate is done using the battery's SoC. At this stage, we will have 2 potential possibilities, either the battery's SoC is appropriate to make a trip from the customer $<\mathrm{i}>$ to customer $\langle\mathrm{j}\rangle$. In this case, the vehicle does not need to visit a charging station. Alternatively, the vehicle must search for a charging station to carry on the tour. 


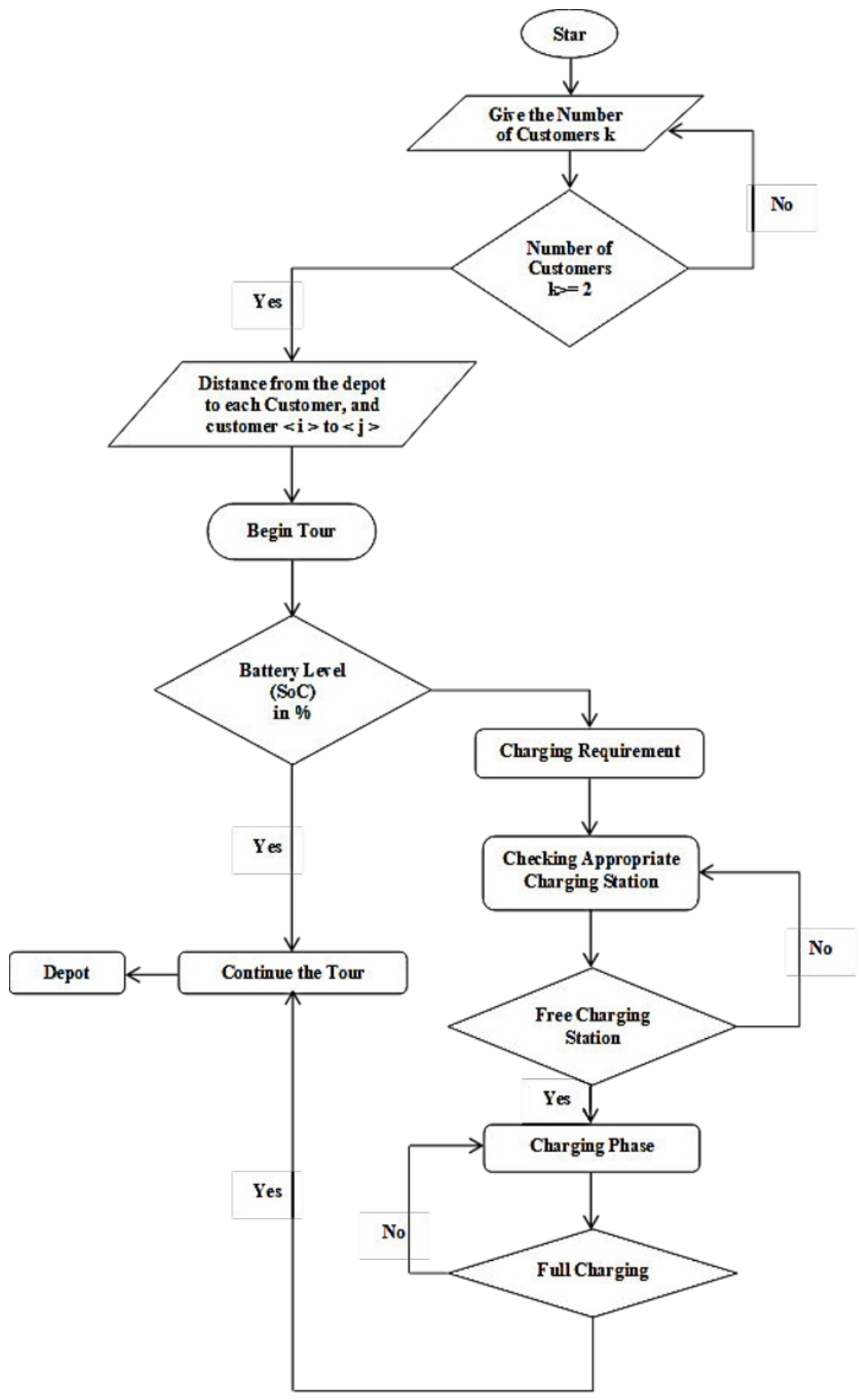

Fig. 7. Diagram of tour optimization. 


\subsection{First results}

The idea of using a purely industrial problem on the GPDP would pave the way for other adaptation methods that promise a variety of solutions. As represented in figure 7, the energy constraint is taken into account based on the Soc of the battery. The average speed of the vehicle is $45 \mathrm{~km} / \mathrm{h}$, the distance between each customer and the depot is given in the table in Kilometres. It is important to note that this calculator can be adapted to any vehicle. For a case study, the following results are provided by a preference of five customers who request to be served.

Table 1. Distance between each customer.

\begin{tabular}{|l|c|c|c|c|c|}
\cline { 2 - 6 } \multicolumn{1}{c|}{} & Customer 1 & Customer 2 & Customer 3 & Customer 4 & Customer 5 \\
\hline Depot & 8 & 3 & 15 & 21 & 1 \\
\hline Customer 1 & & 32 & 14 & 7 & 9 \\
\hline Customer 2 & 32 & & 30 & 35 & 30 \\
\hline Customer 3 & 14 & 30 & & 3 & 12 \\
\hline Customer 4 & 7 & 35 & 3 & & 14 \\
\hline Customer 5 & 9 & 30 & 12 & 14 & \\
\hline
\end{tabular}

Table 2. Distance between each customer and the depot.

\begin{tabular}{|c|c|c|c|c|c|}
\cline { 2 - 6 } \multicolumn{1}{c|}{} & Customer 1 & Customer 2 & Customer 3 & Customer 4 & Customer 5 \\
\hline $\begin{array}{c}\text { Service } \\
\text { Time in } \\
\text { seconds }\end{array}$ & 120 & 120 & 240 & 400 & 320 \\
\hline
\end{tabular}

The overall route traveled to serve the five customers is $53 \mathrm{~km}$ rather than $126 \mathrm{~km}$, which refers to serving the customer 5 first, customer 1 second, customer 4 third, customer 3 fourth, customer 2 fifth and return to the depot. Instead of serving the customer 4 first, customer 5 second, customer 2 third, customer 1 fourth, customer 3 fifth, which corresponds to 5429 seconds rather than 11254 seconds for the whole tour. This time covers each client's service time.

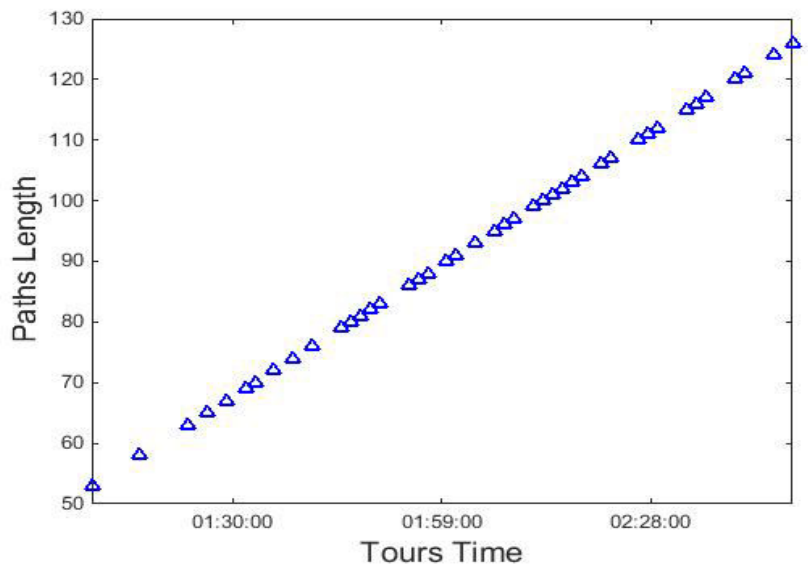

Fig. 8. Possible tours with the corresponding lengths. 
Figure 8 displays the duration of the possible tours with the corresponding lengths. Figures 9 and 10 indicate the variance of the Soc for the optimal and non-optimal choice given by our algorithm. As we compare the two figures, we have served all customers (Figure 9) without passing through a recharging station. However, the choice (Figure 10) will lead to a recharge request at customer 2 . This request would implement an additional time, which is split into time to reach the charging station and time to charging.

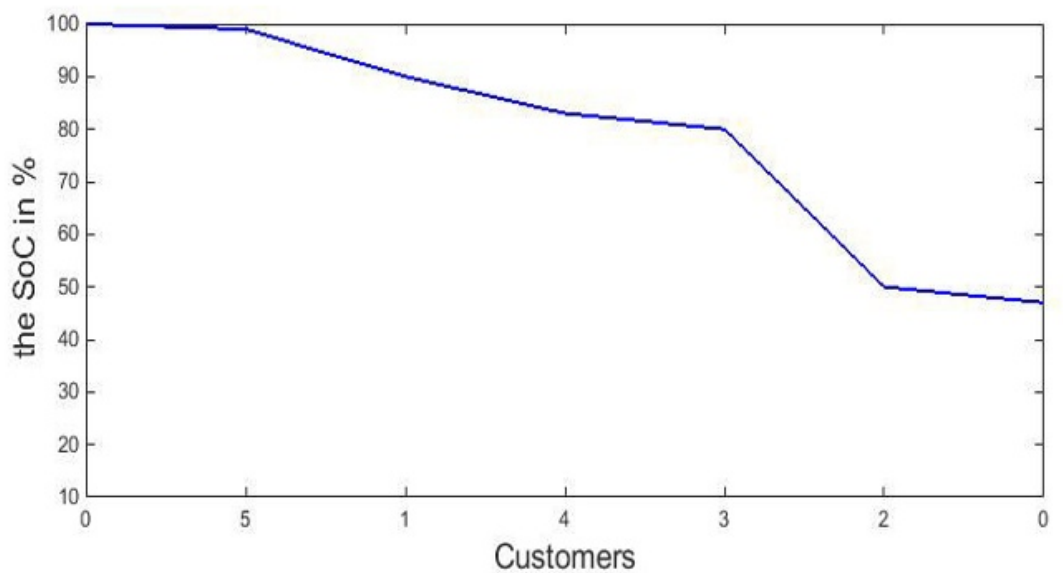

Fig. 9. Variance of the Soc for the optimal choice.

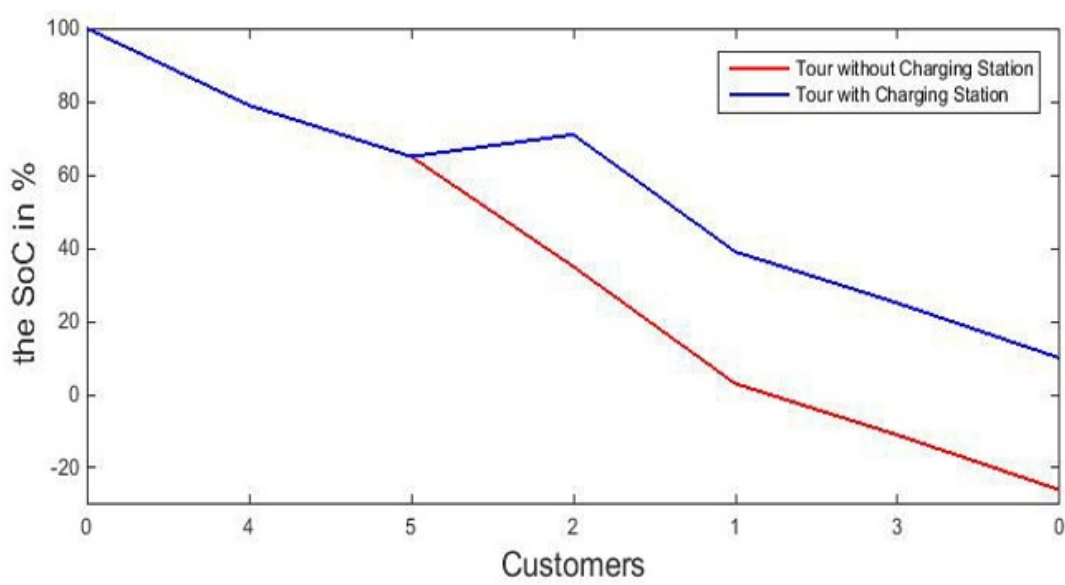

Fig. 10. Variance of the Soc for the non-optimal choice.

\section{Conclusion}

This paper proposed a new concept for addressing pickup and delivery as part of the vehicle routing problem by projecting on an automated manufacturing cell. An optimization approach is developed to minimize the vehicle's energy consumption. This approach will subsequently be based on artificial intelligence through the development of a database that includes all the charging stations, and 
accordingto the first results of this study we can conclude that the reduction of the vehicle's energy consumption is respected.

\section{References}

1. G. Dantzig and R. Ramser. The truck dispatching problem. Management Science, 6:80 - 91, 10 1959.

2. Michel Gendreau, Gilbert Laporte, and Y. Potvin. Metaheuristics for the capacitated vrp. 012001.

3. Cordeau J-F, Laporte G, Mercier A, and Correspondence. A unified tabu search heuristic for vehicle routing problems with time windows.Journal of the Operational Research Society, 52:928 -936, 082001.

4. Eric Taillard, Michel Gendreau, Francois Guertin, and Jean-Yves Potvin. A parallel tabu search heuristic for the vehicle routing problem with time windows. Transportation Research Part Cemerging Technologies - TRANSPORT RES C-EMERG TECHNOL, 5:109 - 122, 041997.

5. Karla Hoffman, Manfred Padberg, and Giovanni Rinaldi. Traveling Salesman Problem, pages 1573 - 1578. 012013.

6. Marc Sol and Martin Savelsbergh. The general pickup and delivery problem. Transportation Science, 29:17 - 29, 021995.

7. Wanyan Yu and Rajan Batta. Chinese Postman Problem. 012011.

8. Bruce Golden and Richard Wong. Capacitated arc routing problem. Networks, 11:305 - 315, 09 1981.

9. Gerardo Berbeglia, Jean-François Cordeau, Irina Gribkovskaia, and Gilbert Laporte. Static pickup and delivery problems: A classification scheme and survey. TOP: An Official Journal of the Spanish Society of Statistics and Operations Research, 15:1 - 31, 022007.

10. Abdelhak Outalha, Yassine Lakhal, Fatima Baghli, and Fouzia Kzaiber. Electric vehicle routing problem using timed petri nets and optimization algorithm. Journal of Advanced Research in Dynamical and Control Systems, 12:1109 - 1115, 052020.

11. Roberto Baldacci, Enrico Bartolini, and Aristide Mingozzi. An exact algorithm for the pickup and delivery problem with time windows. Operations Research, 59:414 - 426, 042011.

12. Haibing Li and Andrew Lim. A metaheuristic for the pickup and delivery problem with time windows. volume 12, pages 160 - 167, 122001.

13. Adria Soriano, Margaretha Gansterer, and Richard Hartl. The tworegion multi-depot pickup and delivery problem. OR Spectrum, 40, 102018.

14. Cornelius Rüther and Julia Rieck. A Grouping Genetic Algorithm for Multi Depot Pickup and Delivery Problems with Time Windows and Heterogeneous Vehicle Fleets, pages 148 - 163. 04 2020.

15. Angel Felipe, M.Teresa Ortuño, Giovanni Righini, and Gregorio Tirado. A heuristic approach for the green vehicle routing problem with multiple technologies and partial recharges. Transportation Research Part E: Logistics and Transportation Review, 71:111 - 128, 112014.

16. Jean-François Cordeau and Gilbert Laporte. A tabu search heuristic for the static multi-vehicle dial-a-ride problem. Transportation Research Part B: Methodological, 37:579 - 594, 072003.

17. Gilbert Laporte. The vehicle routing problem: An overview of exact and approximate algorithms. European Journal of Operational Research, 59:345 - 358, 061992.

18. C.G. Cassandras and Stephane Lafortune. Introduction to Discrete Event Systems, page 800. 01 2010.

19. Daniel Silva, Agnelo Vieira, Eduardo Rocha Loures, Marco Busetti, and Eduardo Santos. Dealing with routing in an automated manufacturing cell: A supervisory control theory application. International Journal of Production Research, 49:4979 - 4998, 082011. 